\title{
The Association between Serum Gamma Glutamyl transferase and Fasting Blood Glucose in Chinese People: A 6-Year Follow-up Study
}

Fei Teng

Xuzhou Central Hospital

Peng Lai

Xuzhou Medical University https://orcid.org/0000-0001-7469-244X

Liying Wang

Xuzhou Central Hospital

Xuekui Liu

Xuzhou Central Hospital

Houfa Geng

Xuzhou Central Hospital

Wei Xu

Xuzhou Central Hospital

Yan Ye

Xuzhou Medical University

Jiayi Li

Bengbu Medical College

Jun Liang ( $\nabla$ mwlj521@njmu.edu.cn )

Affiliated Xuzhou Clinical College of Xuzhou Medical University

\section{Research}

Keywords: Gamma-glutamyl transferase, Fasting blood glucose, Impaired fasting glucose regulation, Follow-up study

Posted Date: October 29th, 2021

DOI: https://doi.org/10.21203/rs.3.rs-968607/v1

License: (1) (1) This work is licensed under a Creative Commons Attribution 4.0 International License.

Read Full License 
The Association between Serum Gamma Glutamyl transferase and Fasting Blood Glucose in Chinese People: A 6-Year Follow-up Study

Fei Teng ${ }^{1,2 \#}$, Peng Lai ${ }^{3 \#}$, Liying Wang ${ }^{1 \#}$, Xuekui Liu ${ }^{1}$, Houfa Geng ${ }^{1}$, Wei Xu ${ }^{1}$, Yan $\mathrm{Ye}^{3}$, Jiayi $\mathrm{Li}^{4}$, Jun Liang ${ }^{5^{*}}$

\section{Affiliations:}

${ }^{1}$ Department of Endocrinology, Xuzhou Central Hospital, Xuzhou, Jiangsu, China, 221000

${ }^{2}$ Xuzhou Institute of Medical Sciences, Xuzhou, Jiangsu, China, 221000

${ }^{3}$ Xuzhou Medical University, Xuzhou, Jiangsu, China, 221000

${ }^{4}$ Bengbu Medical College, Bengbu, Anhui, China, 233000

${ }^{5}$ Affiliated Xuzhou Clinical College of Xuzhou Medical University, Xuzhou, Jiangsu, China, 221000

\section{* Corresponding author at:}

Dr. Jun Liang, MD, PhD

Affiliated Xuzhou Clinical College of Xuzhou Medical University, 199\# South Jiefang Road,

Xuzhou, Jiangsu, China, 221000

Telephone: $+86-18952171209$

Fax: $+86-83840486$

E-mail address: mwlj521@njmu.edu.cn 


\begin{abstract}
Objective The aim of this study was to identify the relationships between gamma-glutamyltransferase (GGT) and fasting blood glucose (FBG) during a 6-year follow-up of subjects, and to evaluate if GGT is an independent factor affecting FBG.
\end{abstract}

Methods Total of 1369 individuals from health examination survey at the urban area of Xuzhou, central China, were followed up for 6 years. The patients were divided into four groups according to their baseline GGT levels (in quartiles). The one-way ANOVA method was used to compare the differences of variables with basic line. The relationship between GGT and FBG levels was examined using repeated measurements ANOVA.

Results The grouping of baseline GGT levels influenced changes in blood glucose during 6-year follow-up. In GGT quartile subgroups, the annual mean increase of FBG level shows a positive relationship with baseline GGT level. And this trend was even more pronounced in the highest baseline GGT group. Time course, baseline FBG and GGT groups at different individuals, which together affected the change of FBG level during the follow-up. Considering that baseline FBG may affect subsequent FBG levels, repeated measures ANOVA was used to exclude the effect of this factor. Results showed that different baseline GGT groups were still significantly associated with increased FBG levels during follow-up. GGT is an independent factor affecting FBG level $(\mathrm{p}<0.001)$.

Conclusions The annual mean increase of FBG level shows a positive relationship with baseline GGT level. Higher baseline GGT levels resulted in a faster annual mean increase in FBG. GGT could be used for early detection of impaired fasting glucose regulation (IFG) patients in clinical field.

Key words: Gamma-glutamyl transferase, Fasting blood glucose, Impaired fasting glucose regulation, Follow-up study

\title{
Introduction
}


Gamma-glutamyl transferase (GGT) is a liver enzyme involved in the gamma glutamyl cycle, which is related to glutathione (GSH) synthesis and degradation ${ }^{[1]}$. The role of GGT is maintaining adequate levels of intracellular glutathione and serum GGT has been proposed as a marker of oxidative stress ${ }^{[2]}$. The measurement of circulating GGT activity is widely used for the diagnosis of liver and obstructive biliary diseases and as a marker of alcohol consumption. Aside from its diagnostic uses, GGT has attracted interest mainly for its association with diabetes and metabolic syndrome, cancer, atherosclerosis, and cardiovascular disease ${ }^{[3]}$. Recently, increased serum concentrations of GGT were again shown to predict the development of type 2 diabetes in a longitudinal study from Korea ${ }^{[4]}$. This would suggest that increased GGT concentrations are somehow related to the primary disease mechanism rather than the consequences of hyperglycemia ${ }^{[5]}$.

Despite extensive research focused on the potential relationship between GGT and the risk of T2DM, no consensus has been reached to date ${ }^{[6-8]}$. Our study aimed to identify the relationships between GGT and fasting blood glucose (FBG) during a 6-year follow-up, and evaluate if GGT is an independent risk factor of impaired fasting glucose regulation.

\section{Study population}

The study involved a population-based prospective cohort recruited from health examination survey at the urban area of Xuzhou, central China. This study approved by the Ethics Committee of the Central Hospital of Xuzhou, China and written informed consent was obtained from all participants.1369 subjects with both laboratory results and questionnaire were considered for our study. The exclusion criteria were as follows: diabetes, confirmed or suspected cancer, chronic kidney disease, pregnancy, and previous history of coronary heart disease, participants who suffered from viral hepatitis, hepatic cirrhosis, alcoholics, alcoholic liver disease or other liver disease, alcohol intake $>140$ grams/week for men and $>70$ grams/week for women. They were followed up for six years from 2010 to 2016 and have a yearly health examination in Physical Examination Center of Xuzhou Central Hospital. 
Finally, the prospective analysis included 1067subjects (421 males and 646 females) aged 20-84 years at baseline examination.

\section{Methods}

All the individuals were divided into four groups (Q1, Q2, Q3, Q4) according to their baseline GGT levels (in quartiles). We first conducted a cross-sectional analysis on the healthy physical examination population to explore the relationship between GGT and fasting blood glucose (FBG), age, weight, body mass index, systolic blood pressure (SBP), diastolic blood pressure (DBP), triglycerides (TG), total cholesterol (TC), high-density lipoprotein (HDL) and low-density lipoprotein (LDL). Then, all subjects were followed up regularly and given annual physical examinations by experienced doctors while being educated and urged to live a healthy lifestyle. And then, we analyzed the change of FBG during 6-year follow up according to quartile of baseline GGT level. Finally, we analyzed the relationship between basic GGT subgroups, time course and basic line FBG.

All subjects underwent anthropometric measurements, included height, weight, blood pressure, and body mass index. After sitting for 5 minutes, the SBP and DBP of the right upper arm were measured by a mercury barometer. The arm and heart were at the same level, measured for 3 times, and the average value was taken for analysis. Body mass index (BMI), calculated as weight divided by the squared height in meters, was used as an index of relative weight. The medical examination questionnaire was designed in a unified manner, including general health situation, sex, age, and past medical history, were recorded by qualified doctors. Blood samples were obtained from an antecubital vein in the morning after a 12-h overnight fast. Biochemical indicators were measured by the enzymatic method on a Hitachi 7600 automatic biochemical analyzer (Hitachi Ltd., Tokyo, Japan).

\section{Statistical analyses}

Data management and statistical analysis were performed using SPSS version 24.0 (SPSS, Inc., Chicago, IL). All measurement data were subjected to a normality test and followed a normal distribution. Data were presented as the mean-standard deviation. The patients were divided into four groups according to their baseline 
GGT levels (in quartiles). The one-way ANOVA method was used to compare the differences of variables with baseline. The relationship between GGT levels and FBG levels was examined using repeated measurements ANOVA. All reported $P$ values were two-tailed, and values of $P<0.05$ were considered statistically significant.

\section{Results}

1. Clinical characteristics according to the quartiles of serum GGT

The mean \pm SD age of the participants was $47.49 \pm 9.60$ years and more than half were female (646 of 1067; 60.54\%). Study populations were grouped according to baseline GGT quartile: for men, Q1: GGT<20 IU/L, Q2: 20<GGT<27 IU/L, Q3: $27<$ GGT<42 IU/L, Q4: GGT >42I U/L; for women, Q1: GGT <12 IU/L,Q2: $12<$ GGT $<16$ IU/L, Q3: $16<$ GGT<22 IU/L, Q4: GGT >22 IU/L. The baseline clinical and biochemical characteristics are shown (Table 1). It shows positive relationships between the baseline serum GGT level and age, weight, BMI, SBP, DBP, TC, TG, and LDL cholesterol levels. Only GGT and HDL cholesterol levels shows a negative relationship $(P<0.001)$.

2. FBG changes in baseline and 6-year follow-up

Changes in FBG at baseline and during 6-year follow-up after the subjects was grouped according to quartile categories according to baseline serum GGT levels are shown (Table 2). FBG level at baseline ( $p<0.05)$, 2-year follow-up $(p<0.01)$, 4-year follow-up $(p<0.05)$ and 6-year follow-up $(p<0.01)$ was positively correlated with GGT level. It means that the grouping of baseline GGT levels influenced changes in fasting blood glucose during 6-year follow-up.

Figure 1 shows that during 6-year follow-up, FBG levels were proportional to the duration of follow-up in all baseline GGT quartile subgroup. In four GGT quartile 
subgroups, the annual mean increase of FBG level shows a positive relationship with baseline GGT level, even more pronounced in the highest baseline GGT group (Q4).

3. The interactions between time course, baseline FBG and baseline GGT level

In Table 3, the baseline FBG was used as the covariate in ANOVA with repeated measurements, and it was found that time course was the factor affecting the change of FBG, and there were interactions between time course, baseline FBG and GGT groups at different individuals, which together affected the change of FBG level during the follow-up.

4. GGT is an independent factor affecting FBG level

Considering that baseline FBG may affect subsequent FBG levels, repeated measures ANOVA was used to exclude the effect of this factor. Results in Table 4 shows that different baseline GGT groups were still significantly associated with increased FBG levels during follow-up. It means that GGT is an independent factor affecting FBG level $(p<0.01)$.

\section{Discussion}

It has been shown that serum GGT concentrations are closely related to the occurrence of diabetes in large populations in Korea ${ }^{[9]}$. In our 6-year follow up general population-based study, we demonstrated the association between GGT and FBG in Chinese People. FBG level at baseline and 2-year, 4-year, 6-year follow-up was positively correlated with the baseline GGT level. It means that the grouping of baseline GGT levels influenced changes in blood glucose during 6-year follow-up. In four GGT quartile subgroups, the annual mean increase of FBG level shows a positive relationship with baseline GGT level. Higher baseline GGT levels resulted in a faster annual mean increase in FBG. GGT could be used for early detection of impaired fasting glucose regulation patients in clinical field.

Our current follow-up analysis demonstrated a significant association between baseline GGT level and IFG risk, independent of age, weight, BMI, blood pressure 
and lipids. As we known, liver plays an important role in the regulation of blood glucose levels, especially in the fasting state ${ }^{[10]}$. Thamer C et al ${ }^{[11]}$ elevated serum GGT levels predict glucose intolerance probably via insulin resistance rather than beta-cell dysfunction. This may be primarily related to hepatic insulin resistance. The association observed between elevated hepatic lipids and reduced insulin sensitivity might explain the increased diabetes risk observed in subjects with elevated serum GGT concentrations. In the absence of overt liver disease, elevated serum GGT concentrations may point the clinician to incipient disturbances in the glucose metabolism ${ }^{[12]}$.

Studies have found that early detection of high fasting glucose and active intervention can effectively prevent the occurrence of type 2 diabetes mellitus, which is the key to alleviating the long-term medical burden ${ }^{[13]}$. Therefore, identification of factors associated with the pathogenesis of $\mathrm{T} 2 \mathrm{DM}$ is of great importance for improving screening of the high-risk population and facilitating early prevention strategy. Pre-diabetes is blood glucose consistently elevated above normal levels, but it is not high enough for diabetes diagnosis, characterized by reduced glucose tolerance or impaired fasting glucose (IFG). Studies have shown that individuals with IFG are at a higher risk of subsequent diabetes ${ }^{[14]}$. Our previous study confirmed that serum GGT levels are positively associated with IFG in Chinese adults, independent of other confounding factors ${ }^{[15]}$. Our recent 6-year follow-up study conducted in Chinese to further evaluate the relationship between GGT and fasting glucose. For our present study, during 6-year follow-up, blood glucose levels were proportional to the duration of follow-up in all baseline GGT quartile subgroup. In four GGT quartile subgroups, the annual mean increase of FBG level shows a positive relationship with baseline GGT level. And this trend was even more pronounced in the highest baseline GGT group. GGT was a simple, accurate, and easy to standardize biochemical indicator, widely used in clinical practice. Our results infer that GGT could be used for early detection of IFG patients in clinical field.

Our study suggested that an increase in GGT concentration within its physiological range is a sensitive and early biomarker for the development of diabetes 
[16]. GGT is a liver enzyme involved in the gamma glutamyl cycle, which is related to glutathione (GSH) synthesis and degradation ${ }^{[17]}$. On our opinion, the gamma glutamyl cycle, mediated by GGT, is the most important endogenous antioxidant system ${ }^{[18]}$. Oxidative stress is associated with a number of pathological conditions, such as aging, inflammation, atherosclerosis, and reperfusion injury ${ }^{[19]}$. Oxidative stress can also play a role in the cause and pathophysiology of diabetes ${ }^{[20]}$. We did a prospective study to test the hypothesis that GGT, possibly as a marker of oxidative stress, is a predictor of incident diabetes ${ }^{[21]}$. In addition, since oxidative stress increases with age ${ }^{[22,23]}$, we tested whether the relations between time course and diabetes were modified by the baseline GGT concentration. As a result, FBG level at baseline and 2-year, 4-year, 6-year follow-up were positively correlated with the GGT level. It means that the grouping of baseline GGT levels influenced changes in blood glucose during 6-year follow-up.

This study was a 6-year follow-up research with a large number of subjects. Different from the cross-sectional studies, the follow up study should provide more important information on the relationship between GGT and fasting glucose as observation of IFG incidence is possible. However, there are some limitations to this study. First, our study based on the data from health examination survey at urban area of Xuzhou, central China, cannot represent the whole Chinese population. Second, observation period is only six years and needs a long-term observation. Hence, a more successful research will be achieved if a foothold is gained for a long-term largescale prospective research through a cooperative study between numerous institutions complementing the above-mentioned limitations. Third, there may be a misclassification bias due to the single measurement of fasting glucose, serum GGT and other biomarkers.

In conclusions, our study showed FBG level at baseline and 2-year, 4-year, 6-year follow-up was positively correlated with the GGT level. It means that the grouping of baseline GGT levels influenced changes in blood glucose during 6-year follow-up. An individual with different baseline FBG in different baseline GGT groups may have different FBG level during follow-up than other individuals. In four 
GGT quartile subgroups, the annual mean increase of FBG level shows a positive relationship with baseline GGT level. In addition, as the measurement of serum GGT is easy and convenient, it could be used for early detection of IFG even diabetes patients in the clinical field.

\section{Ethics approval and consent to participate}

This study approved by the Ethics Committee of the Central Hospital of Xuzhou, China and written informed consent was obtained from all participants.

\section{Consent for publication}

We have reviewed the final version of the manuscript and approved it for publication.

\section{Availability of data and material}

We certify that we have participated sufficiently in the work to take public responsibility for the appropriateness of the design and method, the research, analysis, and interpretation of the data. To the best of our knowledge and belief, this manuscript has not been published in whole or in part elsewhere.

\section{Competing interests}

The authors have no conflicts of interests to disclose.

\section{Funding}

This work was sponsored by Jiangsu Provincial Medical Youth Talent (QNRC2016387), Xuzhou Science and Technology Bureau Project (KC19030), National Natural Science Foundation of China Grant Award (81870540).

\section{Auther Contributions}

Fei Teng, Peng Lai, Liying Wang contributed equally to this work and they completed experimental design, physical measurement, follow-up, data analysis and 
manuscript writing. Xuekui Liu, Houfa Geng, Wei Xu, Yan Ye and Jiayi Li participated in experimental design and data collection. Jun Liang funded the entire study.

\section{Acknowledgements}

Not applicable.

\section{References}

[1]. Speisky H, Shackel N, Varghese G, Wade D, Israel Y. Role of hepatic gamma-glutamyltransferase in the degradation of circulating glutathione: studies in the intact guinea pig perfused liver. Hepatology. 1990 May;11(5):843-9.

[2]. Lee DH, Blomhoff R, Jacobs DR Jr. Is serum gamma glutamyltransferase a marker of oxidative stress? Free Radic Res. 2004 Jun;38(6):535-9.

[3]. Ndrepepa G, Colleran R, Kastrati A. Gamma-glutamyl transferase and the risk of atherosclerosis and coronary heart disease. Clin Chim Acta. 2018 Jan;476:130-138.

[4]. Lee DH, Ha MH, Kim JH, Christiani DC, Gross MD, Steffes M, Blomhoff R, Jacobs DR Jr. Gamma-glutamyltransferase and diabetes--a 4 year follow-up study. Diabetologia. 2003 Mar;46(3):359-64.

[5]. Thamer C, Tschritter O, Haap M, Shirkavand F, Machann J, Fritsche A, Schick F, Häring H, Stumvoll M. Elevated serum GGT concentrations predict reduced insulin sensitivity and increased intrahepatic lipids. Horm Metab Res. 2005 Apr;37(4):246-51.

[6]. Ahn HR, Shin MH, Nam HS, Park KS, Lee YH, Jeong SK, Choi JS, Kweon SS.

The association between liver enzymes and risk of type 2 diabetes: the Namwon study. Diabetol Metab Syndr. 2014 Feb 6;6(1):14.

[7]. Kunutsor SK, Abbasi A, Adler AI. Gamma-glutamyl transferase and risk of type II diabetes: an updated systematic review and dose-response meta-analysis. Ann Epidemiol. 2014 Nov;24(11):809-16.

[8]. Lee YS, Cho Y, Burgess S, Davey Smith G, Relton CL, Shin SY, Shin MJ. Serum gamma-glutamyl transferase and risk of type 2 diabetes in the general Korean 
population: a Mendelian randomization study. Hum Mol Genet. 2016 Sep 1;25(17):3877-3886.

[9]. Hong NS, Kim JG, Lee YM, Kim HW, Kam S, Kim KY, Kim KS, Lee DH. Different associations between obesity and impaired fasting glucose depending on serum gamma-glutamyltransferase levels within normal range: a cross-sectional study. BMC Endocr Disord. 2014 Jul 12;14:57.

[10]. Rui L. Energy metabolism in the liver. Compr Physiol. 2014 Jan;4(1):177-97.

[11]. Thamer C, Tschritter O, Haap M, Shirkavand F, Machann J, Fritsche A, Schick F, Häring H, Stumvoll M. Elevated serum GGT concentrations predict reduced insulin sensitivity and increased intrahepatic lipids. Horm Metab Res. 2005 Apr;37(4):246-51.

[12]. Tangvarasittichai S. Oxidative stress, insulin resistance, dyslipidemia and type 2 diabetes mellitus. World J Diabetes. 2015 Apr 15;6(3):456-80.

[13]. Kim MS, Lee DY. Urinary Glucose Screening for Early Detection of Asymptomatic Type 2 Diabetes in Jeonbuk Province Korean Schoolchildren. J Korean Med Sci. 2017 Jun;32(6):985-991.

[14]. Tabák AG, Herder C, Rathmann W, Brunner EJ, Kivimäki M. Prediabetes: a high-risk state for diabetes development. Lancet. 2012 Jun 16;379(9833):2279-90.

[15]. Wang Y, Wu T, Zang X, Liu X, Xu W, Lai P, Wang Y, Teng F, Qiu Q, Geng H, Liang J. Relationship Between Serum Gamma-Glutamyl Transferase Level and Impaired Fasting Glucose Among Chinese Community-Dwelling Adults: A Follow-Up Observation of 6 Years. Metab Syndr Relat Disord. 2021 Mar;19(2):100-106.

[16]. Hong NS, Kim JG, Lee YM, Kim HW, Kam S, Kim KY, Kim KS, Lee DH. Different associations between obesity and impaired fasting glucose depending on serum gamma-glutamyltransferase levels within normal range: a cross-sectional study. BMC Endocr Disord. 2014 Jul 12;14:57.

[17]. Tangvarasittichai S. Oxidative stress, insulin resistance, dyslipidemia and type 2 diabetes mellitus. World J Diabetes. 2015 Apr 15;6(3):456-80. 
[18]. Sreeram M, Suryakar AN, Dani NH. Is gamma-glutamyl transpeptidase a biomarker for oxidative stress in periodontitis? J Indian Soc Periodontol. 2015 Mar-Apr;19(2):150-4.

[19]. Dröge W. Free radicals in the physiological control of cell function. Physiol Rev. 2002 Jan;82(1):47-95.

[20]. Sreeram M, Suryakar AN, Dani NH. Is gamma-glutamyl transpeptidase a biomarker for oxidative stress in periodontitis? J Indian Soc Periodontol. 2015 Mar-Apr;19(2):150-4.

[21]. Lee DH, Ha MH, Kim JR, Gross M, Jacobs DR Jr. Gamma-glutamyltransferase, alcohol, and blood pressure. A four year follow-up study. Ann Epidemiol. 2002 Feb;12(2):90-6.

[22]. Hensley K, Floyd RA. Reactive oxygen species and protein oxidation in aging: a look back, a look ahead. Arch Biochem Biophys. 2002 Jan 15;397(2):377-83.

[23]. Bakker SJ, IJzerman RG, Teerlink T, Westerhoff HV, Gans RO, Heine RJ. Cytosolic triglycerides and oxidative stress in central obesity: the missing link between excessive atherosclerosis, endothelial dysfunction, and beta-cell failure? Atherosclerosis. $2000 \mathrm{Jan} ; 148(1): 17-21$. 
Figures

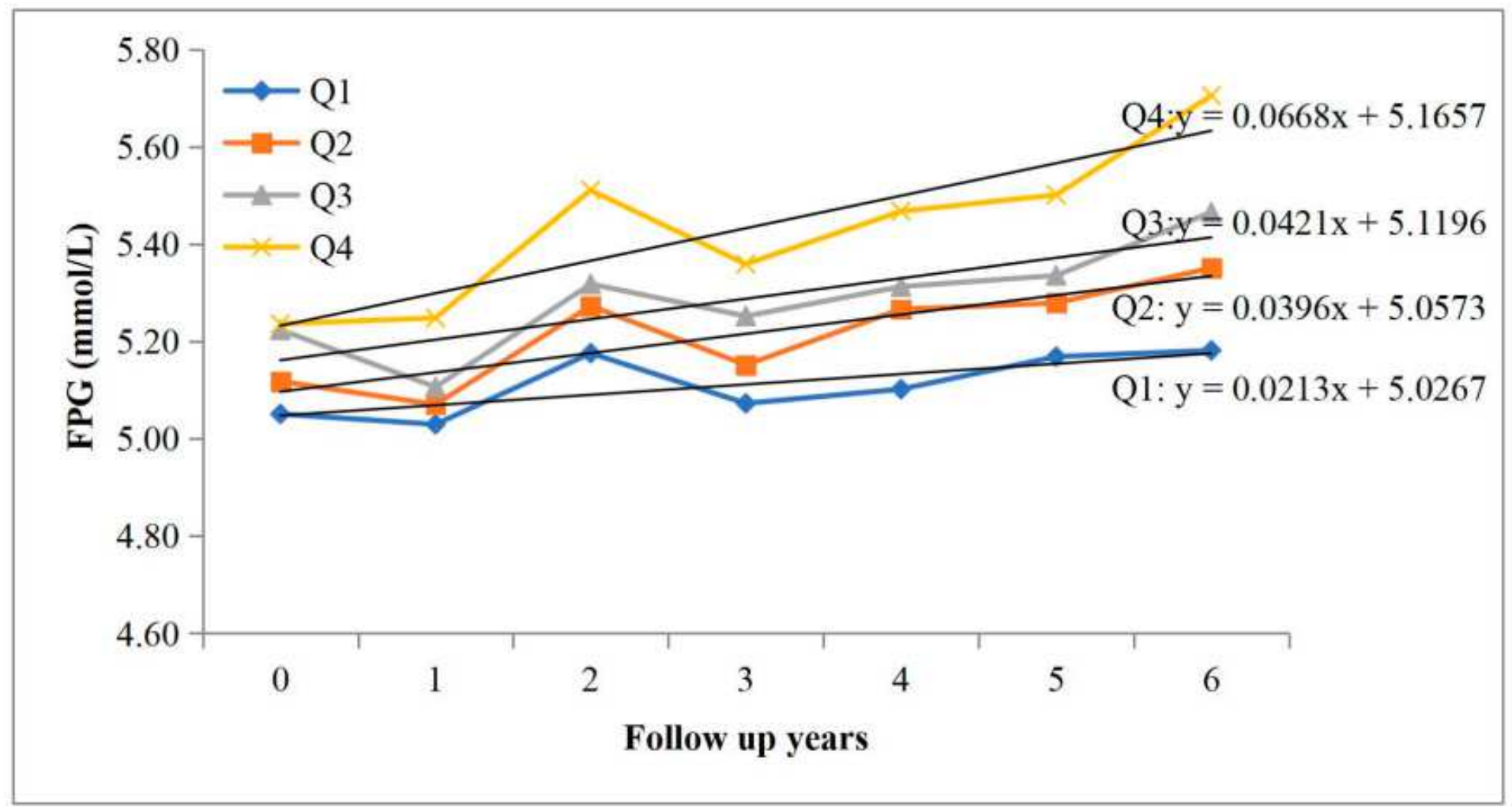

Figure 1

The change of FBG in the follow up study according to quartile of baseline GGT groups.

\section{Supplementary Files}

This is a list of supplementary files associated with this preprint. Click to download.

- Table.pdf 